\title{
The potential of carbonate apatite as an alternative bone substitute material
}

\author{
Ahmad Jabir Rahyussalim, ${ }^{1}$ Sugeng Supriadi, ${ }^{2}$ Aldo Fransiskus Marsetio, ${ }^{1}$ Pancar Muhammad Pribadi ${ }^{2}$, Bambang Suharno ${ }^{2}$
}

pISSN: 0853-1773 • elSSN: 2252-8083 https://doi.org/10.13181/mji.v28i1.2681 Med J Indones. 2019;28:92-7

Received: March 1, 2018

Accepted: January 22, 2019

Author's affiliations:

${ }^{1}$ Department of Orthopaedic and Traumatology, Faculty of Medicine, Universitas Indonesia, Cipto Mangunkusumo Hospital, Jakarta, Indonesia, ${ }^{2}$ Department of Mechanical Engineering, Faculty of Engineering, Universitas Indonesia, Depok, Indonesia

Corresponding author:

Aldo Fransiskus Marsetio

Department of Orthopaedic and

Traumatology, Faculty of Medicine,

Universitas Indonesia, Cipto

Mangunkusumo Hospital, Jalan Salemba

6, Senen, Central Jakarta 10320, DKI

Jakarta, Indonesia

Tel/Fax: +62-21-3155996/

+62-21-3929655

E-mail: aldofransiskus@gmail.com

\begin{abstract}
Bone reconstructive surgery has become a common procedure, and bone transplantation has become the second most frequently performed tissue transplantation procedure worldwide. Therefore, the need for bone substitute materials has increased. Artificial bone substitutes exhibit osteoconductive properties and feature several advantages, including abundant resources, low cost, and low donor site morbidity. Carbonate apatite ( $\left.\mathrm{CO}_{3} \mathrm{Ap}\right)$ is a calcium phosphate ceramic that can be used as a synthetic bone graft. The carbonate content of this ceramic is similar to that of bone apatite. In this review, we show that carbonate apatite can be degraded given its chemical reactivity in a weakly acidic environment and through osteoclast resorption. Moreover, it is osteoconductive and promotes bone tissue formation without fibrotic tissue formation. Additionally, microstructural analysis revealed that new bone tissue is formed within the bone graft itself.
\end{abstract}

KEYWORDS bone graft, bone substitute, bone scaffold, calcium phosphate, carbonate apatite, orthopedic biomaterials
The number of bone reconstructive surgeries has progressively increased worldwide. Every year, over two million bone grafting procedures are performed globally. The need for bone substitutes has increased because bone transplantation has become the second most frequently performed tissue transplantation procedure in the world. ${ }^{1}$ In orthopedics, bone grafts are often needed in cases of trauma, oncology, prosthesis revision, and spine surgery. ${ }^{2}$ Bone substitute materials must be biocompatible, biodegradable, bioresorbable, and osteoconductive. ${ }^{3}$ Bone grafts can be mainly classified as autografts, allografts, and xenografts.

Autogenous bone grafts are the golden standard for bone grafting given their properties of osteoconductivity, osteoinductivity, and biocompatibility, which is defined as the ability to promote osteogenesis with minimal immunological reaction. ${ }^{4,5}$ Nevertheless, bone harvesting has several drawbacks and is associated with morbidity. ${ }^{6}$ Autogenous bone grafts are commonly collected from the iliac bone and the proximal part of the tibia of the patients themselves. These sources, however, are finite. Thus, their use in the reconstruction of large bone defects is inappropriate. Additionally, the creation of incisions in healthy skin and the partial destruction of bone often prolong postoperative pain. Therefore, a readily available alternative to synthetic bone grafts is preferred to reduce postoperative morbidity.

Calcium phosphate ceramics, collagen, bioactive glasses, and biodegradable polymers are some types 
of synthetic bone substitutes that have been studied or used for the clinical treatment of bone defects. Hydroxyapatite (HAp) and $\beta$-tricalcium phosphate are calcium phosphate ceramics that have been widely applied for the reconstruction of lost bone tissue in orthopedic surgery. ${ }^{7}$ These bone substitutes act as scaffolds and have the potential to induce bone formation within their pores. ${ }^{8}$

Although HAp shows excellent biocompatibility and good osteoconductivity, its key drawback is its stability as a foreign substance in the bone. Ayukawa et al9 found that HAp granules were enclosed by fibrous tissue at 2 weeks after implantation. HAp has lower osteoconductivity than carbonate apatite granules. These findings show that HAp poses a risk for secondary infection given its difficult resorption in the bone defect.

In contrast to HAp, $\beta$-tricalcium phosphate is resorbed well by macrophages and osteoclasts and promotes collagen fiber deposition. ${ }^{10}$ Animal studies have shown that this process could take up to 6 months. ${ }^{11}$ However, another study found that $\beta$-tricalcium phosphate is only partially absorbed and its use as a bone graft is associated with several complications, such as inflammation and bone cyst formation. ${ }^{12,13}$

\section{Innate response toward synthetic bone grafts}

Human bone undergoes remodeling in real time. Remodeling is a physiological process wherein old, primary, damaged, or ischemic bone is replaced and calcium homeostasis is maintained. This process is dependent on two main cells, namely, osteoclasts and osteoblasts. Osteoclasts are multinucleated cells that resorb the bone matrix, whereas osteoblasts are osteogenic cells that form the new bone matrix. These cells work in concert to maintain the balance between bone resorption and bone formation. ${ }^{14}$

Giannoudis et $\mathrm{al}^{15}$ proposed the diamond concept, which states that four elements are essential for fracture healing (Figure 1). These four elements include osteogenic cells, osteoinductive growth factors, osteoconductive scaffolds, and mechanical stability. Osteogenic bone marrow cells are mesenchymal stem cells that, along with growth factors, induce a cellular event cascade that initiates and stimulates healing. Scaffolds are extracellular matrixes that provide an osteoconductive environment and promotes cellular interactions, adhesion, and growth. Stable mechanical fixation is also essential for fracture healing.

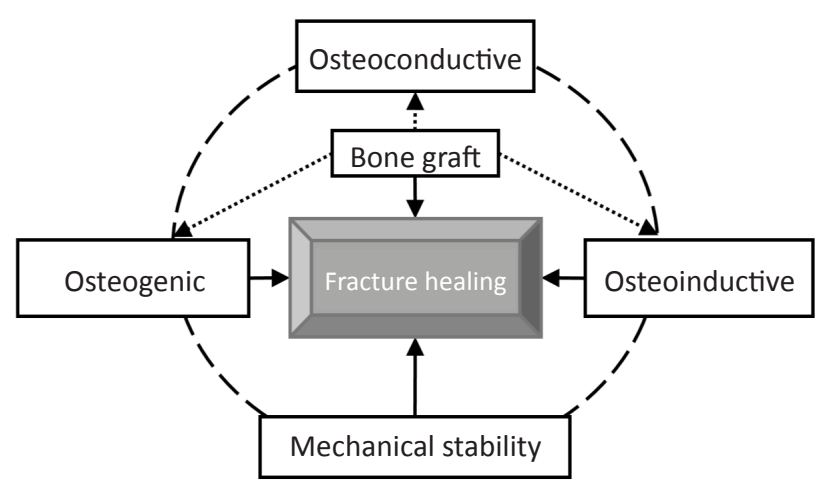

Figure 1. Role of bone grafting in fracture healing. Bone grafts are osteoconductive, osteoinductive, and osteogenic. These properties, in addition to mechanical stability, fulfill the diamond concept of bone healing

However, nonideal conditions for spontaneous bone healing are unavoidable. Modern internal fixation alone cannot overcome infection, poor vascularization, malnutrition, or bone defects. Bone grafts are used to augment bone healing by providing a supporting platform and osteoconductive and osteoinductive factors that promote bone remodeling. Upon implantation, the bone graft will undergo integration in concert with bone remodeling. Fibrin blood clots form around materials and bone marrow cells are recruited onto the surfaces of the biomaterial upon the release of growth factors and biological signals. ${ }^{16}$ After the surge of inflammatory cells, the mesenchymal stem cells of the bone assimilate with the graft site. Osteoinductive factors then induce these cells to differentiate into chondroblasts and osteoblasts. Afterward, the bone graft is enclosed, revascularized, and resorbed by new osteoclast cells. The graft will serve as a three-dimensional framework (scaffold) if it is not resorbed. The contributing factors that determine bone graft resorption include cell origin, environmental $\mathrm{pH}$, sintering temperature and duration, porosity, surface roughness, and grain size.

New capillary ingrowth, perivascular tissue, and mesenchymal stem cells are passively induced by the osteoconductive properties of the bone graft. Mesenchymal stem cells and growth factors for bone healing are also induced by the osteoinductive properties of the bone graft. Examples of these cells and factors include bone morphogenetic proteins (BMP)-2, -4, and -7; platelet-derived growth factors; interleukins; fibroblast growth factors (FGFs); granulocyte-macrophage colony-stimulating factors; and angiogenic factors, such as vascular endothelial 
growth factors. ${ }^{17}$ Mesenchymal stem cells, osteoblasts, and osteocytes are the main osteogenic components of bone grafts and facilitate new bone formation.

\section{Main characteristics of carbonate apatite}

Carbonate apatite has the general formula of $\mathrm{CO}_{3} \mathrm{Ap}$. It is a natural inorganic component of human bone tissue. The general formula of apatite is $\mathrm{M}_{10}\left(\mathrm{ZO}_{4}\right)_{6} \mathrm{X}_{2}$. Two known types of carbonate apatite exist and are differentiated on the basis of the substitution of their $\mathrm{CO}_{3}^{2-}$ components. Carbonate apatite type $A$ is synthesized through the substitution of $\mathrm{CO}_{3}{ }^{2-}$ on the $\mathrm{X}$ position (hydroxyl ion $\left[\mathrm{OH}^{-}\right]$) at high temperature. Carbon apatite type $\mathrm{B}$, the most ubiquitous biological carbonate apatite, forms through $\mathrm{CO}_{3}{ }^{2-}$ substitution on the $\mathrm{ZO}_{4}$ position (phosphate ion $\left[\mathrm{PO}_{4}{ }^{3-}\right]$ ) at low temperature. The carbonate content of carbon apatite depends on processing type and can reach 4-8 wt.\%, which is similar to that of bone (4-6 wt.\%). ${ }^{18}$

Although carbonate apatite can be regarded as bone apatite, its powder form cannot be used directly as a bone substitute. The direct use of the powder forms of carbonate apatite as a bone substitute would induce inflammatory response upon implantation. Inflammatory responses, in turn, would result in crystal formation. Apatite must be fabricated into granular blocks or granular ceramics via sintering. However, carbonate apatite often undergoes decomposition during sintering because of the high temperatures used in the process. ${ }^{18}$

\section{Development of carbonate apatite as a synthetic bone graft}

Composition, processing, and morphology must be considered in the design and fabrication of appropriate bone graft materials. Carbonate apatite cannot be used in its powder form because it results in inflammatory reaction-induced crystal formation. Carbonate apatite must be processed into blocks through sintering, which involves high temperatures. An earlier study revealed that carbonate specimens with 12 wt.\% carbonate content can be sintered at low temperatures between $600^{\circ} \mathrm{C}-750^{\circ} \mathrm{C} .{ }^{19}$ The specimens retained approximately $6 \mathrm{wt} . \%$ of latticesubstituted carbonate in a single apatitic phase mass. The carbonate content retained by these specimens was higher than that retained by bone apatite (approximately $4-8 \mathrm{wt} . \%$ ).
A follow-up study revealed that the dissolution rate of sintered carbonate apatite in acidic environments was 10 times lower than that of bone apatite. ${ }^{19}$ Bone apatite has low crystallinity and high surface area. These characteristics accelerate the dissolution of bone apatite. However, the reactivity of carbonate apatite in acid dissolution is higher than that of hydroxyapatite (HAp) given the carbonate content of the former in the apatite lattice. Dissolution rate is determined by solution $\mathrm{pH}$, stirring rate, and particle size.

Suh et $\mathrm{al}^{3}$ attempted to process carbonate apatite as a bone graft with type I atelocollagen extracted from bovine tail skins. They first sintered specimens at $980^{\circ} \mathrm{C}$ and $1200^{\circ} \mathrm{C}$ and then performed ultraviolet (UV) and dehydrothermal (DHT) technique to increase the number of collagen fibrillary crosslinks in the specimens. Specimens sintered at $980^{\circ} \mathrm{C}$ and treated with UV or through DHT maintained favorable physicochemical and biological properties. This study also revealed that poor cellular response was caused by recrystallization and reductions in carbonate ion content.

The compositional transformation reaction based on the dissipation-precipitation reaction proposed by Ana et $\mathrm{al}^{20}$ is the latest alternative for the fabrication of carbonate apatite in block shapes. The dissolution-precipitation reaction is similar to the gypsum formation process. In this reaction, a portion of the precursor phase is dissolved in liquid, and its precipitates form the final product. Carbonate apatite is more thermodynamically stable than HAp in a physiological environment (weak acid; $\mathrm{pH}$ 7.4). Thus, materials with a moderate level of solubility must be selected as the precursor phase. Such materials include calcium carbonate; calcium phosphate; and phosphate compounds, such as zinc phosphate. ${ }^{9,18}$

Matsuura et $\mathrm{al}^{21}$ attempted to identify the optimal carbonate content of bone grafts. They found that over long-term observation (24 weeks after implantation), the new bone formation area ratio obtained with grafts with 6 wt.\% carbonate was higher than obtained with grafts with different carbonate contents. This result may be attributed to the similarity between the carbonate content of the graft and that of bone (4-6 wt.\%). They suggested that carbonate apatite with a carbonate content of $6 \mathrm{wt} . \%$ is suitable for bone grafts. 


\section{Physiological response of cells and tissues to carbonate apatite}

One study revealed that bone graft materials with carbonate contents of 1-3 wt.\% are highly soluble and that solubility increases dramatically over this range of carbonate content. Carbonate apatite is thermodynamically more stable and less soluble than HAp. Sintered carbonate apatite is highly soluble under acidic environmental conditions ( $\mathrm{pH}$ 5.0). Specifically, under acidic environmental conditions, carbonate apatite dissolves within $30 \mathrm{~s}$, whereas sintered HAp dissolves within 3.8 days. ${ }^{19}$ Carbonate apatite shows high dissolution capability in the physiological environment ( $\mathrm{pH}$ 7.4). The resorbability of carbonate apatite by osteoclasts is higher than that of sintered HAp in physiological or acidic environments. ${ }^{18}$ The high chemical reactivity in weak acids and resorption capability by bone tissue of carbonate apatite can be attributed to the carbonate content within the apatite lattice. ${ }^{3}$

The responses of osteoblasts to carbonate apatite can be used as an indicator of osteoconductivity because it determines the turnover of materials within bone tissue. ${ }^{18}$ Ayukawa et al ${ }^{9}$ found that in contrast to HAp, carbonate apatite can intensively bind with bone tissue without inducing fibrotic tissue formation. By contrast, Nagai et al $^{16}$ found no difference in cell count until the $7^{\text {th }}$ day. Nevertheless, carbonate apatite exerted drastically different effects on the expression patterns of the differentiation markers of type I collagen, alkaline phosphatase, osteopontin, and osteocalcin and improved the regulation of osteoblast cell differentiation. The remodeling process triggered by carbonate apatite resembles the natural bone remodeling process given the high osteoconductivity and similarity of carbonate apatite with bone tissue on the cellular level.

This finding is supported by a recent study that applied micro-CT scanning to evaluate bone defects in rabbits at 18 months after reconstruction with carbonate apatite granules made from dicalcium phosphate dihydrate blocks. This study found that after 24 weeks of implantation, carbonate apatite was resorbed and replaced by new bone tissue, whereas HAp was not replaced by bone tissue. The resorption rate of carbonate apatite implanted at the metaphysial of the proximal tibia was twice than carbonate apatite implanted at the epiphysis of the distal femur. Given that the resorption rate of carbonate apatite is linear, complete resorption was estimated to occur at 1-1.5 years after implantation. ${ }^{22}$

Habibovic et $\mathrm{al}^{23}$ developed two types of carbonate apatite ceramics and tested their capability to support bone healing. The first sample (CA-A) was sintered at $900^{\circ} \mathrm{C}$ and had a carbonation level of 3 wt.\% and large pores. The second sample (CA-B) was sintered at $700^{\circ} \mathrm{C}$ and had a carbonation level of $5 \mathrm{wt} . \%$, small grains, and micropores. The specific surface area of CA-B was 10 times larger than that of CA-A. Bone formation at the implantation area of $C A-A$ was higher than that at the implantation area of CA-B. Moreover, the dissolution of $C A-A$ was more pronounced than that of CA-B. In addition, the induction of ectopic bone formation by CA-A was earlier than that by CA-B. Although CA-A had good osteoconductive properties, it lost its osteoinductive capacity because of its rapid dissolution. Thus, CA-A did not facilitate bridging despite promoting good bone ingrowth given its rapid surface degradation.

Suh et $\mathrm{al}^{3}$ processed carbonate apatite with atelocollagen type I. They then sintered the material at $980^{\circ} \mathrm{C}$ or $1200^{\circ} \mathrm{C}$ and processed it with UV or through DHT. They found that specimens sintered at $980^{\circ} \mathrm{C}$ and treated with UV or through DHT showed good cell adhesion, cell proliferation, and alkaline phosphatase activity. Moreover, the materials maintained their physicochemical and biological properties.

Keiichi et $\mathrm{al}^{24}$ observed the supplementation effect of basic FGFs loaded on disk-shaped porous carbonate apatite. Carbonate apatite disks were made by mixing carbonate apatite powder with commercial sugar (grain size of $500-850 \mu \mathrm{m}$ ). The mixture was compacted, immersed in distilled water at $37^{\circ} \mathrm{C}$, dried at room temperature overnight, and then sintered at $700^{\circ} \mathrm{C}$ for $1 \mathrm{~h}$. Stable, porous carbonate apatite grains with sizes of $0.3^{-1} \mu \mathrm{m}$ and pore sizes of $0.5^{-1.5} \mu \mathrm{m}$ were obtained. Micro-CT analysis revealed that although new bone formation was seen in both groups, growth was drastically higher in the basic fibroblast growth factor (bFGF)-loaded group.

Nagai et $\mathrm{al}^{16}$ attempted to combine bone morphogenetic protein-2 with carbonate apatite granules processed through the dissolutionprecipitation technique. They revealed that $50 \mu \mathrm{g}$ of bone morphogenic protein-2 (BMP-2) can stimulate new bone formation by promoting the osteoblastic differentiation of mesenchymal cells in the surrounding tissue. By contrast, no new bone formation was 
observed in the group treated with only $5 \mu$ g of BMP-2 and or without BMP-2. Material resorption decreased by $30 \%$ of the initial value, and residual tartrateresistant acid phosphatase (TRAP)-positive cells were observed.

Nagai et al $^{16}$ attempted to observe the behavior of osteoblast cells upon carbonate apatite implantation. They observe that the rates of the initial attachment and proliferation of human bone marrow cells on HAp were similar to those on carbonate apatite but were lower than those on the tissue culture plate control. Moreover, the ability of carbonate apatite to promote osteoblastic differentiation was better than that of HAp, as shown by the increased expression level of human bone marrow cells differentiation markers, which included the markers of early osteogenesis (collagen type I (COL-1) and alkaline phosphatase) and late osteogenesis (osteopontin and osteocalcin). The elevated expression of latestage markers in human bone marrow cells cultured on carbonate apatite suggests that carbonate apatite can support the differentiation of cells into mature osteoblasts. Moreover, human bone marrow cells cultured on carbonate apatite underwent osteoblastic differentiation earlier than those cultured on HAp.

\section{Clinical applications of carbonate apatite}

Carbonate apatite may be superior to other kinds of artificial bone substitute. It meets the basic requirements for a bone graft material: osteoconductivity, osteoinductivity, and the ability to promote osteogenesis with minimal immunological reaction. Additionally, carbonate apatite has similar carbon content as human bone tissue. It is highly resorbable and can be replaced by bone tissue. It can induce osteoblast responses and can promote osteoblast differentiation well. The results of earlier studies suggest that carbonate apatite may be used as an alternative for artificial bone grafts. However, clinical trials on the use of carbonate apatite as a bone graft has only reached the stage of animal trials. $9,21,22,24$ Only one study on the application of carbonate apatite in humans exists. In this study, carbonate apatite was used in mixed cement form for craniofacial reconstruction. ${ }^{25}$ However, studies on the utilization of carbonate apatite in long bones remain unreported likely because carbonate apatite remains scarce and expensive.

\section{Limitation}

The orthopedic uses of carbonate apatite have several limitations. A major weakness of carbonate apatite is its weak mechanical property. ${ }^{26}$ It is brittle and has poor fatigue resistance. The brittleness and poor fatigue resistance of carbonate apatite are related to its large pore diameters. Carbon apatite, however, is suitable as a drug carrier and has a large surface area given its large pores. It is also rapidly soluble given its large pores and low crystallinity. ${ }^{19}$

\section{Suggestions for future studies}

Future works should include in vivo studies with human subjects. Moreover, they should focus on adjusting the solubility of carbonate apatite through the application of coatings or the formation of composites with other materials. Future works should also investigate the capability of this material to fill bone defects, the development of bioactive implant coatings and biodegradable antibiotic-impregnated scaffolds, and many other possibilities.

\section{Conclusion}

Carbonate apatite is a good alternative for bone graft materials given its good osteoclast resorbability, osteoconductivity, osteoinductivity, and osseointegration. It is also an excellent bone scaffold. Many aspects of carbonate apatite warrant exploration and improvement.

\section{Conflict of Interest}

The authors declare that there is no conflict of interest regarding the publication of this paper.

\section{Acknowledgment}

This literature review is part of our study, which is funded by International Indexed Publications for Student Final Project grant by Universitas Indonesia and Decentralization and Competitive grant by Indonesian Ministry of Research Technology and Higher Education.

\section{Funding Sources}

This study was funded by International Indexed Publications for Student Final Project (PITTA) grant.

\section{REFERENCES}

1. Campana V, Milano G, Pagano E, Barba M, Cicione C, Salonna $G$, et al. Bone substitutes in orthopaedic surgery: from basic science to clinical practice. J Mater Sci Mater Med. 2014;25(10):2445-61.

2. Bhatt RA, Rozental TD. Bone graft substitutes. Hand Clin 2012;28(4):457-68.

3. Suh H, Park JC, Han DW, Lee DH, Han CD. A bone replaceable artificial bone substitute: cytotoxicity, cell adhesion, proliferation, and alkaline phosphatase activity. Artif Organs. 2001;25(1):14-21. 
4. Giannoudis PV, Dinopoulos H, Tsiridis E. Bone substitutes: an update. Injury. 2005;36 Suppl 3:S20-7.

5. Moore WR, Graves SE, Bain GI. Synthetic bone graft substitutes. ANZ J Surg. 2001;71(6):354-61.

6. Taylor BC, French BG, Fowler TT, Russell J, Poka A. Induced membrane technique for reconstruction to manage bone loss. J Am Acad Orthop Surg. 2012;20(3):142-50.

7. Yuasa T, Miyamoto Y, Ishikawa K, Takechi M, Momota Y, Tatehara S, et al. Effects of apatite cements on proliferation and differentiation of human osteoblasts in vitro. Biomaterials. 2004;25(7-8):1159-66.

8. Kasai T, Sato K, Kanematsu Y, Shikimori M, Kanematsu N, Doi $\mathrm{Y}$. Bone tissue engineering using porous carbonate apatite and bone marrow cells. J Craniofac Surg. 2010;21(2):473-8.

9. Ayukawa Y, Suzuki Y, Tsuru K, Koyano K, Ishikawa K. Histological comparison in rats between carbonate apatite fabricated from gypsum and sintered hydroxyapatite on bone remodeling. Biomed Res Int. 2015;2015:579541.

10. Chazono M, Tanaka T, Kitasato S, Kikuchi T, Marumo K. Electron microscopic study on bone formation and bioresorption after implantation of beta-tricalcium phosphate in rabbit models. J Orthop Sci. 2008;13(6):550-5.

11. Horowitz RA, Mazor Z, Foitzik C, Prasad H, Rohrer M, Palti A. $\beta$-tricalcium phosphate as bone substitute material. J Osseointegration. 2010;1(2):61-8.

12. Friesenbichler J, Maurer-Ertl W, Sadoghi P, Pirker-Fruehauf U, Bodo K, Leithner A. Adverse reactions of artificial bone graft substitutes: lessons learned from using tricalcium phosphate geneX(R) ${ }^{\circledR}$. Clin Orthop Relat Res. 2014;472(3):976-82.

13. Ogose A, Hotta T, Kawashima H, Kondo N, Gu W, Kamura T, et al. Comparison of hydroxyapatite and beta tricalcium phosphate as bone substitutes after excision of bone tumors. J Biomed Mater Res B Applied Biomater. 2005;72(1):94-101.

14. Rucci N. Molecular biology of bone remodelling. Clin Cases Miner Bone Metab. 2008;5(1):49-56.

15. Giannoudis PV, Einhorn TA, Marsh D. Fracture healing: the diamond concept. Injury. 2007;38 Suppl 4:S3-6.

16. Nagai H, Kobayashi-Fujioka M, Fujisawa K, Ohe G, Takamaru
$\mathrm{N}$, Hara K, et al. Effects of low crystalline carbonate apatite on proliferation and osteoblastic differentiation of human bone marrow cells. J Mater Sci Mater Med. 2015;26(2):99.

17. Khan SN, Cammisa FP Jr, Sandhu HS, Diwan AD, Girardi FP, Lane JM. The biology of bone grafting. J Am Acad Orthop Surg. 2005;13(1):77-86.

18. Ishikawa K. Carbonate apatite bone replacement. In: Antoniac IV, editor. Handbook of Bioceramics and Biocomposites. 1st ed: Springer; 2016. p. 213-32.

19. Doi Y, Shibutani T, Moriwaki Y, Kajimoto T, Iwayama Y. Sintered carbonate apatites as bioresorbable bone substitutes. J Biomed Mater Res. 1998;39(4):603-10.

20. Ana I, Matsuya S, Ishikawa K. Engineering of carbonate apatite bone substitute based on composition-transformation of gypsum and calcium hydroxide. Eng. 2010;2(5):344-52.

21. Matsuura A, Kubo T, Doi K, Hayashi K, Morita K, Yokota R, et al. Bone formation ability of carbonate apatite-collagen scaffolds with different carbonate contents. Dent Mater J. 2009;28(2):234-42.

22. Kanazawa M, Tsuru K, Fukuda N, Sakemi Y, Nakashima Y, Ishikawa K. Evaluation of carbonate apatite blocks fabricated from dicalcium phosphate dihydrate blocks for reconstruction of rabbit femoral and tibial defects. J Mater Sci Mater Med. 2017;28(6):85.

23. Habibovic P, Juhl MV, Clyens S, Martinetti R, Dolcini L, Theilgaard $\mathrm{N}$, et al. Comparison of two carbonated apatite ceramics in vivo. Acta Biomater. 2010;6(6):2219-26.

24. Keiichi K, Mitsunobu K, Masafumi S, Yutaka D, Toshiaki S. Induction of new bone by basic FGF-loaded porous carbonate apatite implants in femur defects in rats. Clin Oral Implants Res. 2009;20(6):560-5.

25. Mathur KK, Tatum SA, Kellman RM. Carbonated apatite and hydroxyapatite in craniofacial reconstruction. Arch Facial Plast Surg. 2003;5(5):379-83.

26. Gómez-Morales J, lafisco M, Delgado-López JM, Sarda S, Drouet C. Progress on the preparation of nanocrystalline apatites and surface characterization: overview of fundamental and applied aspects. Prog Cryst Growth Ch. 2013;59(1):1-46. 